\title{
Utilidad de Bcl-2 como nuevo marcador de expresión de células basales en patología prostática
}

\author{
Ramos Soler D, Mayordomo Aranda E, Calatayud Blas A, Rubio Briones J*, Solsona Narbón E*, \\ Llombart Bosch A.
}

Departamento de Patología. Facultad de Medicina. Universitat de Valencia.
*Fundación Instituto Valenciano de Oncología (IVO). Valencia.

"Beca Pedro Cifuentes Díaz". Fundación para la Investigación en Urología (FIU).

Actas Urol Esp 2006; 30 (4): 345-352

\section{RESUMEN}

\section{UTILIDAD DE BCL-2 COMO NUEVO MARCADOR DE EXPRESIÓN DE CÉLULAS BASALES EN PATOLOGÍA PROSTÁTICA}

Introducción y objetivos: El diagnóstico de adenocarcinoma invasor de próstata es con frecuencia difícil en biopsias cilíndricas, siendo fundamental en estos casos la determinación de la presencia de células basales. En la actualidad, la expresión inmunohistoquímica del antígeno 34betaE12 junto con la proteína p63 son los marcadores más utilizados. En nuestro estudio, se analizó comparativamente la expresión de 34betaE12, p63, bcl-2 y alfa-metilacil-CoA racemasa para valorar la utilidad de bcl-2 como nuevo marcador de células basales en patología prostática.

Métodos y resultados: El estudio incluía piezas de prostatectomía de 48 pacientes en los que se analizó la ausencia de marcaje inmunohistoquímico en el compartimento basal de áreas de adenocarcinoma invasor junto a la expresión de racemasa. Se comprobó también la presencia de células basales en áreas de atrofia, hiperplasia, adenosis y neoplasia intraepitelial prostática (PIN) de alto grado, observándose en ocasiones una expresión discontinua de dichos marcadores en las áreas de adenosis y PIN. En 2 de los 48 casos (4,2\%) de carcinoma se obtuvo no obstante una expresión débil de bcl-2 aunque sin un patrón de distribución basal. Además, la expresión de bcl-2 en los linfocitos del estroma resultó esencial como control interno positivo de la técnica.

Conclusiones: Nuestro estudio demuestra el valor diagnóstico de bcl-2 como nuevo marcador de células basales añadido a los marcadores clásicos.

Palabras clave: Cáncer próstata. Bcl-2. Inmunohistoquímica. Células basales.

\section{ABSTRACT}

USEFULNESS OF BCL-2 EXPRESSION AS A NEW BASAL CELL MARKER IN PROSTATIC PATHOLOGY

Introduction and objectives: The diagnosis of invasive adenocarcinoma of the prostate is often difficult in needle prostatic cores, where, additionally, the assessment of the presence of basal cells has demonstrated to be of paramount importance. Currently, the immunohistochemical expression of 34 betaE12 antigen and p63 protein are the most utilized markers. In our study, we analyzed comparatively the expression of 34betaE12, p63, bcl-2 and alpha-methylacyl-CoA racemase in order to evaluate the usefulness of bcl-2 as a new marker of the basal cells in prostatic pathology.

Methods and results: This study comprises radical prostatectomy specimens from 48 patients which were studied in order to determine the lack of staining of basal cells in invasive tumor areas together with the expression of racemase. Likewise, the presence of basal cells in areas of atrophy, hyperplasia, adenosis, and high-grade prostatic intraepithelial neoplasia (PIN) was also examined. Within the areas of adenosis and PIN a discontinuous pattern of basal cell expression was found in some cases. In 2 out of 48 cases $(4,2 \%)$ of invasive carcinoma a weak bcl-2 expression without a basal cell distribution was found. Moreover, the expression of bcl-2 in the stromal lymphocytes appeared to be essential as an internal positive control of the technique.

Conclusions: In addition to classical markers, we demonstrated the diagnostic value of bcl-2 as a new basal cell marker.

Keywords: Prostate cancer. Bcl-2. Immunohistochemistry. Basal cells. 
$\mathrm{E}^{1}$ diagnóstico diferencial entre adenocarcinoma invasor de próstata y las distintas lesiones proliferativas microglandulares atípicas es con frecuencia complicado y supone además un reto para el patólogo en el diagnóstico de rutina sin la utilización de otras técnicas complementarias. En concreto, en el año 1953, Totten et al. describieron por primera vez la ausencia de células basales en los adenocarcinomas infiltrantes de las glándula prostática ${ }^{1}$. Es por ello que se han estudiado diferentes marcadores inmunohistoquímicos que faciliten la identificación del compartimento basal de las glándulas prostáticas y que indiquen, de esta forma, la naturaleza benigna del proceso, o bien que demuestren una total ausencia de estas células basales en el caso de que se trate de una lesión epitelial maligna con un carácter infiltrante ${ }^{1-2}$.

Entre los diferentes marcadores que se han utilizado hasta la fecha merece la pena destacar la expresión inmunohistoquímica de citoqueratinas de alto peso molecular (antígeno 34betaE12) y de la proteína 03 por parte de las células basales del epitelio prostático ${ }^{3-6}$, así como la expresión de alfa-metilacil-CoA racemasa por parte de las células epiteliales malignas en el caso del adenocarcinoma invasor de próstata y la neoplasia intraepitelial prostática (PIN) de alto grado ${ }^{7-11}$. Junto con estos marcadores se han descrito recientemente otros como es el caso del antígeno S-100A6, c-erbB-2 y kalicreína $4^{12-15}$.

En el presente estudio se pretende demostrar la utilidad de bcl-2 como nuevo marcador de células basales en patología prostática, así como analizar sus posibles ventajas y desventajas en comparación con la expresión inmunohistoquímica de los marcadores clásicos descritos previamente en la literatura: p63, 34betaE12 y racemasa.

\section{MATERIAL Y MÉTODOS}

Datos clínico-patológicos

Nuestro estudio incluyó un total de 48 pacientes con un diagnóstico de adenocarcinoma invasor de próstata con grados sumados de Gleason comprendidos entre 6 y 8 . Todos los pacientes fueron diagnosticados e intervenidos en el Servicio de Urología de la Fundación Instituto Valenciano de Oncología (FIVO). Tras la interven- ción quirúrgica, las piezas de prostatectomía radical fueron fijadas en formaldehído tamponado al $10 \%$ e incluidas a continuación en parafina para llevar a cabo el procesado histológico de las muestras y la posterior tinción de rutina de hematoxilina y eosina (H-E). En todos los casos, un uropatólogo experto seleccionó aquellas secciones mejor conservadas que incluían en el mismo corte histológico el mayor número de áreas de interés para el presente estudio: adenocarcinoma invasor, PIN de alto grado, hiperplasia adenomatosa atípica (adenosis), atrofia, e hiperplasia leiomioadenomatosa de próstata.

\section{Estudio inmunohistoquímico}

Este estudio se realizó sobre cortes histológicos de 4 micras de espesor de bloques incluidos en parafina según el método de la $\mathrm{ABC}$-peroxidasa descrito por Hsu et al. (1981) ${ }^{16}$. Los anticuerpos monoclonales utilizados fueron: citoqueratinas (CKs) de alto peso molecular (clon 34betaE12), bcl-2 (clon 124), p63 (clon 4A4), alfametilacil-coA racemasa (clon 13H4), datos que aparecen todos ellos reflejados en la Tabla 1 . Previamente todas las muestras fueron tratadas mediante calentamiento en autoclave para la recuperación antigénica (1,5 ATM durante 3 min.) y en solución de tampón citrato a pH:6. En la expresión inmunohistoquímica de citoqueratinas de alto peso molecular (citoqueratinas 1, 5, 10 y 14) y de bcl-2 se consideró en ambas una positividad citoplasmática de las células basales del epitelio prostático, mientras que la expresión de p63 se determinó en esas mismas células basales a nivel nuclear. En cuanto a la expresión de alfa-metilacil-CoA racemasa, se valoró la expresión citoplasmática de tipo granular en las células tumorales, tanto en las áreas de PIN de alto grado como en las áreas de adenocarcinoma invasor.

En las áreas lesionales anteriormente descritas se valoró en cada uno de los casos la intensidad de la reacción inmunohistoquímica frente al antígeno 34betaE12, bcl-2 y racemasa de la siguiente forma: 1+, débil; 2+, moderada; 3+, intensa. De igual forma, se registraron aquellos casos de atrofia glandular donde se evidenció un patrón de expresión luminal (no basal) predominante. En las áreas de PIN de alto grado y de ade- 
nosis se consideraron igualmente aquellas zonas donde existía un marcaje basal de tipo discontinuo. Es de destacar que en nuestro estudio un marcaje específico del estrato basal (tanto de forma continua como discontinua) fue considerado siempre como sinónimo de lesión prostática benigna o no invasiva (Tabla 1). En el caso de p63 no se valoró la intensidad de la reacción inmunohistoquímica al tratarse de un antígeno de localización exclusivamente nuclear.

Tabla 1

Condiciones de los anticuerpos primarios utilizados

\begin{tabular}{lccc}
\hline Ac (clon) & Casa C. & Pre-tratamiento & Dilución \\
\hline $\begin{array}{l}\text { CKs A.P.M } \\
\text { (34betaE12) }\end{array}$ & $\begin{array}{c}\text { DAKO } \\
\text { Citrato / M }\end{array}$ & Autoclave & \\
P63 & DAKO & Autoclave & $1 / 50$ \\
$(4 \mathrm{~A} 4)$ & Citrato / M & & \\
$\begin{array}{l}\text { BCL-2 } \\
(124)\end{array}$ & $\begin{array}{c}\text { DAKO } \\
\text { Citrato / M }\end{array}$ & Autoclave & $1 / 50$ \\
$\begin{array}{l}\text { Racemasa } \\
(13 \mathrm{H} 4)\end{array}$ & $\begin{array}{c}\text { DAKO } \\
\text { Citrato / M }\end{array}$ & Autoclave & \\
\hline
\end{tabular}

CKs A.P.M, citoqueratinas de alto peso molecular.

\section{RESULTADOS}

El estudio histopatológico de los 48 pacientes de nuestro estudio incluía finalmente 44 casos $(91,6 \%)$ de hiperplasia benigna de próstata (HBP), $42(87,5 \%)$ de atrofia, 7 lesiones microglandulares atípicas "tipo adenosis" (14,58\%), 38 (79,16\%) que incluían áreas de PIN de alto grado, y 48 (100\%) con diagnóstico de adenocarcinoma infiltrante de próstata. Las áreas de PIN de bajo grado no fueron incluidas en el estudio. En los 48 casos de adenocarcinoma invasor el grado sumado de Gleason fue: Gleason 6 en 27 casos (56,26\%), Gleason 7 en 13 casos $(27,08 \%)$, y Gleason 8 en los 8 casos restantes $(16,66 \%)$.

Los resultados globales de nuestro estudio reflejados en la Tabla 2 demostraron una neta delimitación del compartimento basal de las glándulas prostáticas con los 3 marcadores especí-

Tabla 2 ficos de células basales utilizados (34betaE12, p63 y bcl-2), siendo el marcaje inmunohistoquímico más intenso con el cóctel de citoqueratinas de alto peso molecular (antígeno 34betaE12), seguido de bcl-2 y finalmente de la proteína p63 (Fig. 1A-C).

Dentro de las áreas de hiperplasia y atrofia se identificaron zonas con una importante hiperplasia de células basales, las cuales se pusieron de manifiesto de forma destacada con los distintos marcadores de células basales utilizados en nuestro estudio, incluida la expresión de bcl-2 (Fig. 1D). Es de destacar así mismo la presencia de un patrón de expresión basal discontinuo en las áreas de atrofia y PIN de alto grado con los 3 marcadores de células basales. En estos casos, la expresión nuclear p63 de forma discontinua explicaría las dificultades que encontramos en la identificación del estrato basal, situación que no ocurre en el caso de bcl-2 (Fig. 2A-D). En algunas zonas de atrofia se observó con relativa frecuencia una pérdida completa o parcial del estrato luminal en el epitelio glandular (Fig. 1A-D).

En las zonas de adenocarcinoma invasor se constató una ausencia total de células basales (ausencia de expresión de los 3 marcadores específicos) (Fig. 3A), aunque un reducido porcentaje de los casos estudiados $(4,17 \%)$ presentó un marcaje débil y focal frente a bcl-2. Sin embargo, la expresión de bcl-2 no seguía en ningún caso el

Resultados globales del estudio inmunohistoquímico.

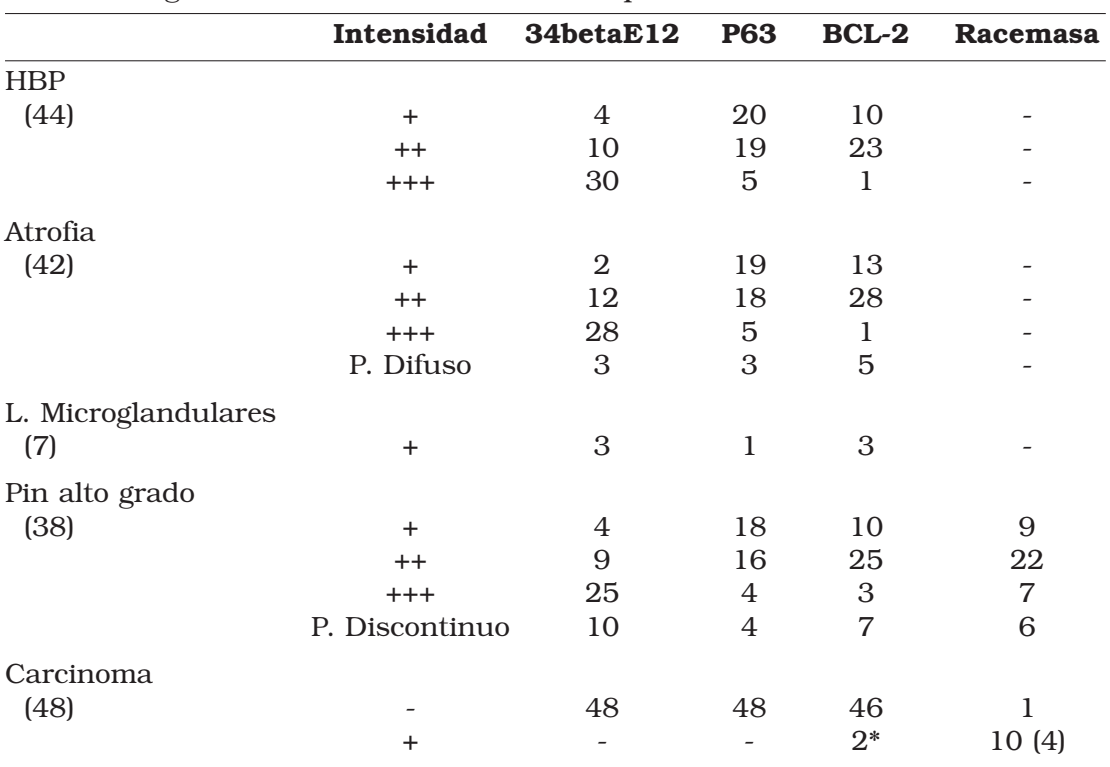

H.B.P, Hiperplasia benigna próstata. * Expresión débil y focal de Bcl-2. 

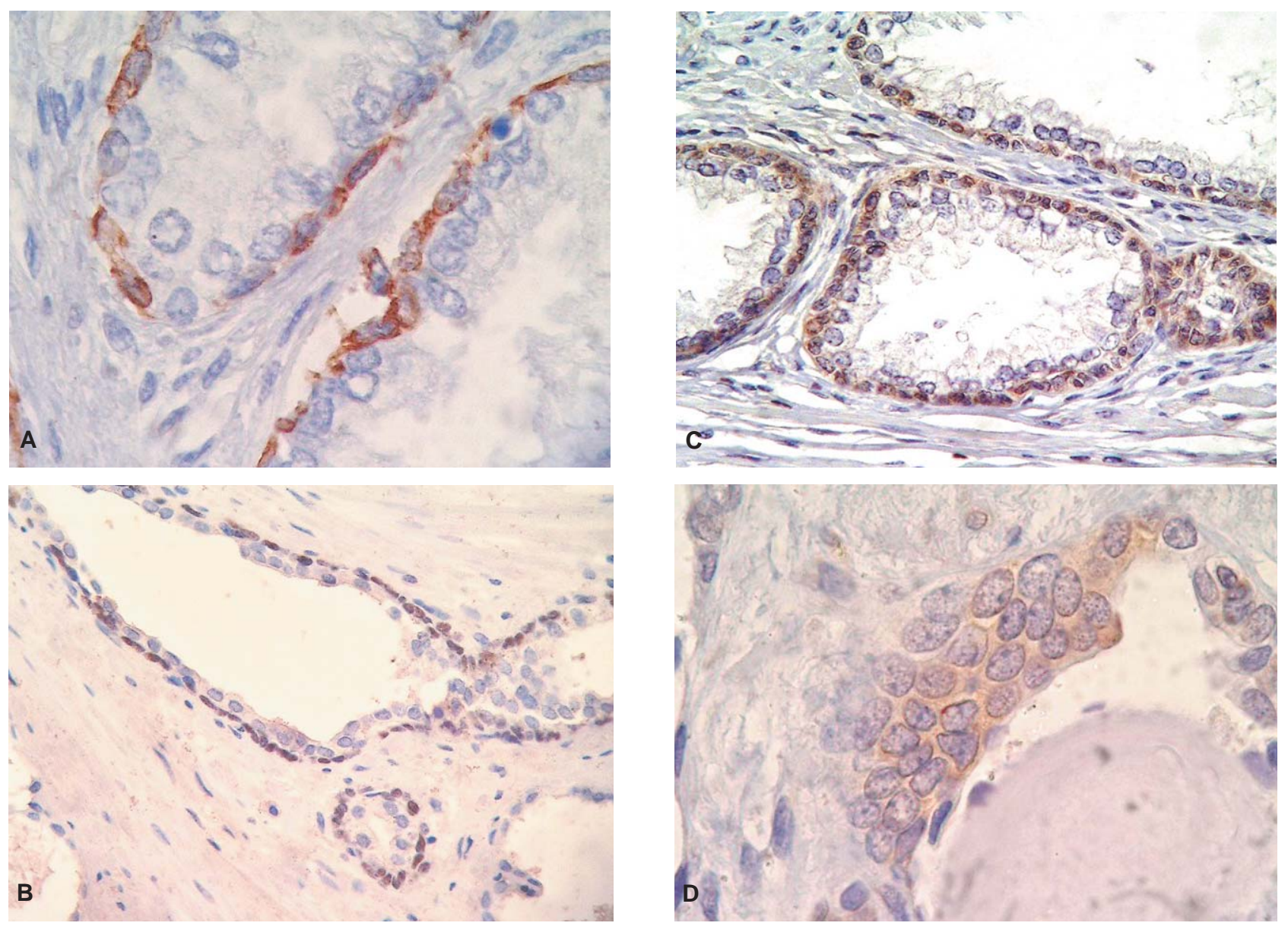

FIGURA 1. Delimitación inmunohistoquímica del compartimento basal en lesiones prostáticas benignas (A: citoqueratina 34betaE12; B: P63; C: Bcl-2) y un caso de hiperplasia de células basales (D: Bcl-2).

característico patrón de distribución basal. Un hallazgo de interés fue también la identificación en los linfocitos del estroma de reactividad frente a bcl-2, lo cual resultó muy útil en nuestra serie como control interno positivo de la técnica inmunohistoquímica (Fig. 3B).

En cuanto a la expresión de alfa-metilacil-CoA racemasa se pudo comprobar una reactividad citoplasmática granular de intensidad muy variable según los distintos casos y en las diferentes áreas lesionales dentro de los mismos (Fig. 4A-B). Excepcionalmente, en 1 de los 48 casos $(2,1 \%)$ se pudo observar una absoluta negatividad frente a la racemasa en las áreas de adenocarcinoma invasor, comprobado este hecho mediante la ausencia total de células basales en esa misma zona con los otros 3 marcadores. Por otro lado, en 8 de los 28 casos $(28,57 \%)$ en los que se observó una reactividad heterogénea (discontinua) frente a la racemasa se pudo comprobar una expresión débil y focal con este mismo marcador. No obstante, en casi la mitad de los casos $(40,4 \%)$ la reactividad de las áreas de adenocarcinoma invasor frente a racemasa resultó muy intensa y continua en la mayor parte de glándulas tumorales. Resultados muy similares en cuanto a marcaje débil y discontinuo con la racemasa se observaron en prácticamente los mismos casos en las áreas de PIN de alto grado en estrecha relación con áreas de adenocarcinoma invasor.

\section{DISCUSIÓN}

La demostración de la ausencia de células basales en el epitelio prostático ha resultado ser determinante en el diagnóstico de malignidad de las lesiones microglandulares atípicas de la próstata, mientras que la presencia de dicho compartimento basal (ya sea de forma continua o discontinua) indicaría probablemente que se trata de una lesión proliferativa benigna microglandular simu- 

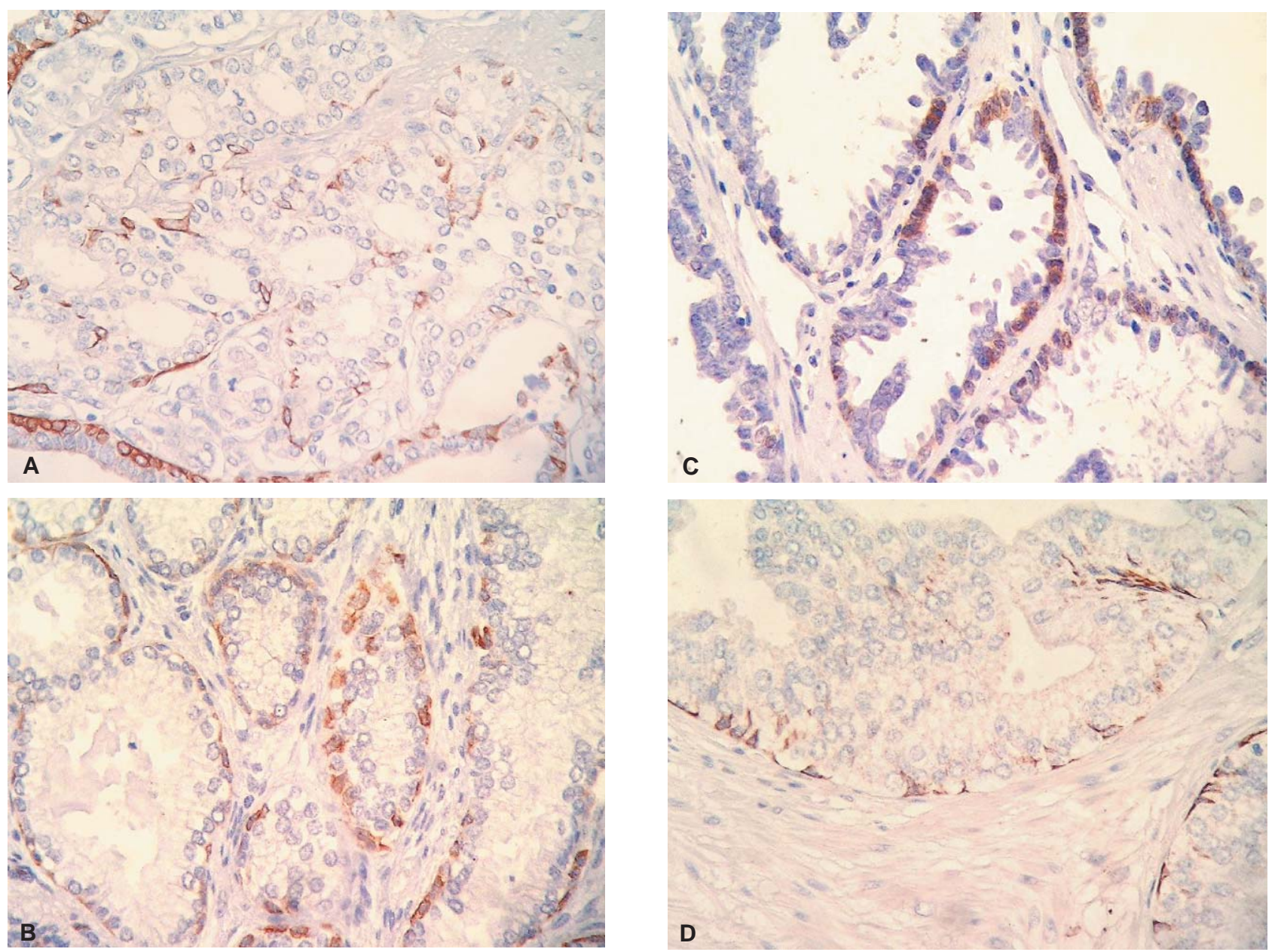

FIGURA 2. Patrón de expresión discontinuo en casos de adenosis (A: citoqueratina 34betaE12; B: Bcl-2) y en casos de PIN de alto grado (C: Bcl-2; D: citoqueratina 34betaE12).

ladora de adenocarcinoma invasor ${ }^{1}$. Es por ello que la determinación de la ausencia de expresión inmunohistoquímica frente a citoqueratinas de alto peso molecular (antígeno 34betaE12) y de la proteína p63 han llegado a ser en la actualidad los marcadores de elección en el diagnóstico diferencial de estas lesiones junto con el hallazgo de la expresión de alfa-metilacil-CoA racemasa por parte de las glándulas tumorales malignas ${ }^{16}$, como se comentará en detalle a continuación. La utilidad de estos marcadores específicos en la detección del estrato basal de las glándulas prostáticas ha sido probada en numerosos estudios previos, tanto de forma individual ${ }^{3}$ como mediante la utilización de cócteles de anticuerpos ${ }^{5,6,8}$ que incluyen a varios de estos marcadores simultáneamente y demuestran en general una mayor sensibilidad y especificidad en la discriminación de este tipo de lesiones sospechosas de malignidad.
En las diferentes series estudiadas en la literatura, la expresión de bcl-2 por parte de las células tumorales ha sido descrita como inhibidor de los procesos de apoptosis y ha demostrado un indudable valor pronóstico. En estas mismas series se describe además una expresión débil y focal de este marcador en determinados casos de PIN de alto grado y de adenocarcinoma invasor ${ }^{17-20}$, aunque en ningún caso se observa un patrón de distribución basal. En el presente estudio, la expresión inmunohistoquímica frente a bcl-2 ha demostrado de igual forma ser un marcador específico en la detección de células basales en patología prostática, con un poder de discriminación en relación con la intensidad de la reacción inferior al de las citoqueratinas de alto peso molecular, pero netamente superior a la expresión nuclear de la proteína p63. Además, y como valor añadido a la fiabilidad del resultado 


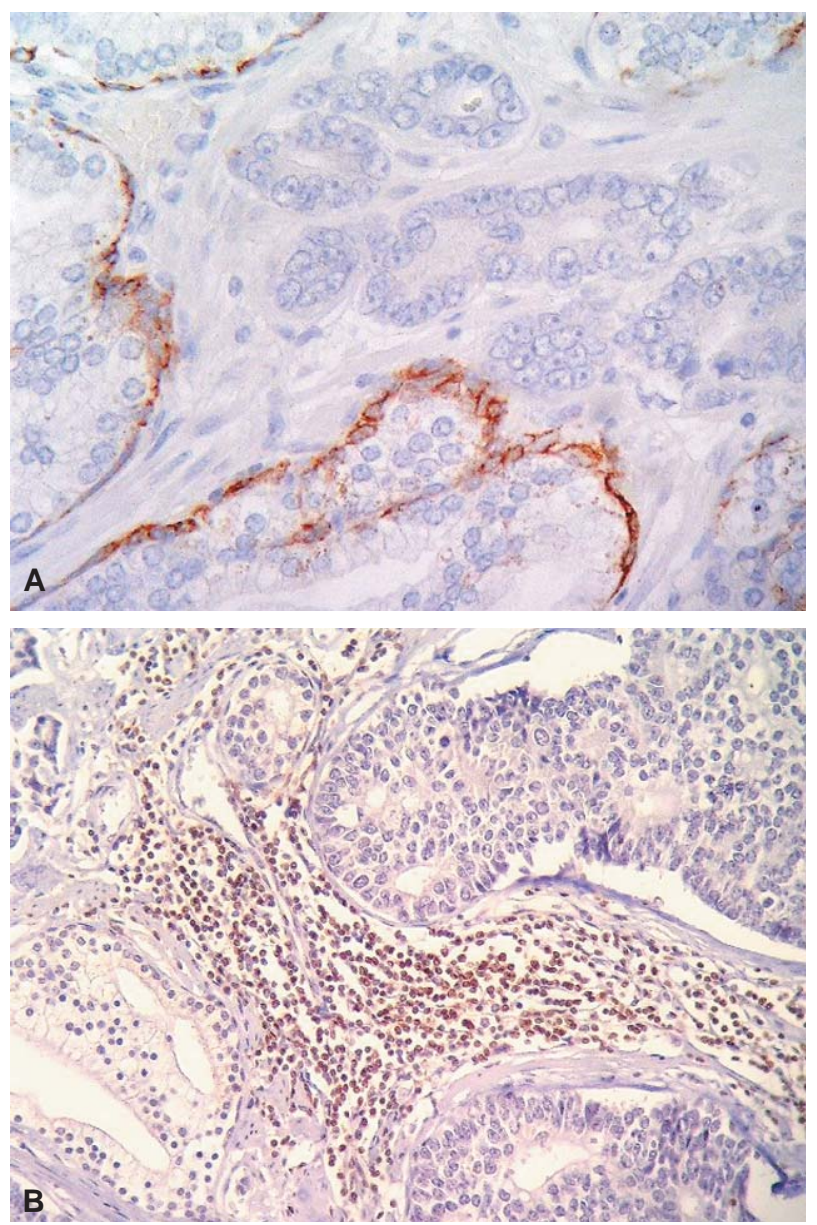

FIGURA 3. Ausencia de expresión inmunohistoquímica frente a citoqueratinas de alto peso molecular (A) y Bcl-2 (B) en las glándulas neoplásicas invasivas. Obsérvese la presencia de reactividad frente a Bcl-2 en los linfocitos del estroma (B).

negativo de la prueba, cabe señalar que la expresión intensa de bcl-2 en los linfocitos del estroma tumoral descrita con anterioridad por Wang et al. $(2004)^{21}$ podría ser utilizada en el futuro como control interno positivo de la técnica inmunohistoquímica, lo cual mejoraría la fiabilidad del resultado, sobre todo en biopsias cilíndricas. Por tanto, nuestro estudio refuerza la posibilidad de introducir bcl-2 como un nuevo elemento integrante de los cócteles de anticuerpos para la detección de células basales en lesiones sospechosas de malignidad en cilindros prostáticos y en piezas de prostatectomía.

En diferentes estudios se describe una expresión inmunohistoquímica de los marcadores de células basales de forma discontinua o heterogénea en las áreas de PIN de alto grado y de adenosis, lo cual supone una dificultad añadida al diagnóstico correcto de las lesiones sospechosas de malignidad $^{22,23}$. En nuestro estudio se confirmó de forma fehaciente esa reactividad de tipo discontinuo en las áreas de adenosis y de PIN, e incluso en áreas de atrofia epitelial, donde las mayores dificultades en la identificación del compartimento basal las encontramos en nuestra experiencia con la expresión nuclear de la proteína p63. Estos 3 marcadores específicos en la detección de células basales (34betaE12, p63 y bcl-2) resultaron ser bastante específicos en la delimitación de las áreas de hiperplasia de células basales dentro de las áreas de hiperplasia simple y de atrofia glandular.

La expresión de alfa-metilacil-CoA racemasa ha sido recientemente descrita como un nuevo marcador positivo de adenocarcinoma invasor de próstata y de sus lesiones precursoras (PIN de alto grado) ${ }^{6-10}$. En nuestro estudio se demostró una ausencia total de reactividad frente a la racemasa

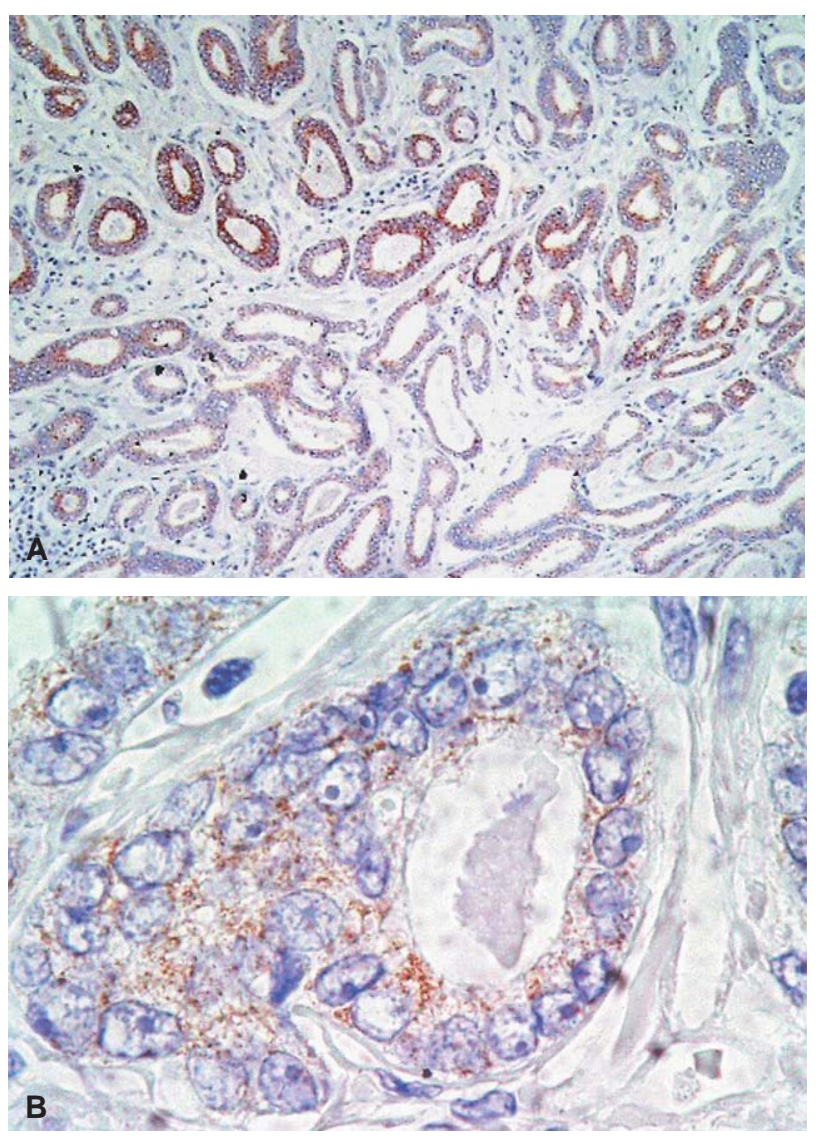

FIGURA 4. Expresión heterogénea de alfa-metilacil-CoA racemasa $(A)$ con un carácter granular citoplasmático (B). 
en las glándulas benignas adyacentes al tumor, a diferencia de Ananthanarayanan et al. (2005) ${ }^{9}$, los cuales sí describen este hallazgo de forma convincente. De igual forma, se observó una total ausencia de reactividad frente a racemasa en las áreas de atrofia, hiperplasia y de adenosis, observándose por el contrario una expresión variable de este marcador en las áreas de PIN de bajo y alto grado y de adenocarcinoma invasor. Previamente, Farinola y Epstein (2004) ${ }^{11}$ describieron la expresión de racemasa en algunos casos de atrofia prostática en relación con áreas de carcinoma. En nuestra serie, sin embargo, no se pudo confirmar este hallazgo, salvo en un caso en el que la positividad frente a racemasa fue débil y focal en las zonas de atrofia en estrecha relación con áreas de adenocarcinoma invasor del subtipo atrófico. No obstante, es de destacar la ausencia total de expresión de este marcador en uno de los casos de nuestro estudio, así como la ausencia focal de expresión de alfa-metilacil-CoA racemasa en los casos de adenocarcinoma invasor de la variante histológica de células claras (Gleason 4), hallazgo que ya fue descrito con anterioridad por Zhou et al. (2003) ${ }^{24}$. Esta heterogeneidad en la expresión de la racemasa ha sido ampliamente descrita en la literatura previa y supone a nuestro juicio una importante limitación al uso individual de este marcador en el diagnóstico de las lesiones sospechosas de malignidad, lo cual se confirmó también en nuestro estudio ${ }^{25}$.

\section{CONCLUSIONES}

En comparación con los marcadores clásicos de células basales, la expresión de bcl-2 ha demostrado de igual forma un indudable valor en el diagnóstico diferencial de las lesiones microglandulares atípicas de la glándula prostática, y aporta como novedad interesante la expresión de bcl2 en los linfocitos del estroma, lo cual podría ser utilizado como control positivo de la técnica inmunohistoquímica en biopsias cilíndricas y en piezas de prostatectomía.

\section{REFERENCIAS}

1. Totten RS, Heinemann MW, Hudson PB, Sproul EE, Stout AP. Microscopic differential diagnosis of latent carcinoma of prostate. AMA Arch 1953;55:131-141.

2. Bonkhoff $\mathrm{H}$. Role of the basal cells in premalignant changes of the human prostate: a stem cell concept for the development of prostate cancer. Eur Urol 1996;30:201-205.
3. Shah RB, Lakshmi PK, Shen R, LeBlanc M, Zhou M, Rubin MA. Usefulness of basal cell cocktail (34betaE12 + p63) in the diagnosis of atypical prostate glandular proliferation. Am J Clin Pathol 2004;122:517-523.

4. Wu HH, Lapkus O, Corbin M. Comparison of 34 betaE12 and p63 in 100 consecutive prostate carcinoma diagnosed by needle biopsies. Appl Immunohistochem Mol Morphol 2004;12:285-289.

5. Hameed O, Sublett J, Humphrey PA. Immunohistochemical stains for p63 and alpha-methylacyl-CoA racemase, versus a cocktail comprising both in the diagnosis of prostatic carcinoma: a comparison of the immunohistochemical staining of 430 foci in radical prostatectomy and needle biopsy tissues. Am J Surg Pathol 2005;29:579-587.

6. Jiang Z, Li C, Fischer A, Dresser K, Woda BA. Using an AMACR (P504S/34betaE12/p63 cocktail for the detection of small focal prostate carcinoma in needle biopsy specimens. Am J Clin Pathol 2005;123:231-236.

7. Zhou M, Aydin H, Kanane H, Epstein JI. How often does alpha-methylacyl-CoA-racemase contribute to resolving an atypical diagnosis on prostate needle biopsy beyond that provided by basal cell markers? Am J Surg Pathol 2004;28:239-243.

8. Molinie V, Herve JM, Lebret T, Lugagne-Delpon PM, Saporta F, Yonneau L, et al. Value of the antibody cocktail anti p63+anti p504S for the diagnosis of prostatic cancer. Ann Pathol 2004;24:6-16.

9. Ananthanarayanan V, Deaton RJ, Yang XJ, Pins MR, Gann $\mathrm{PH}$. Alpha-methylacyl-CoA racemase (AMACR) expression in normal prostatic glands and high-grade intraepithelial neoplasia (HGPIN): association with diagnosis of prostate cancer. Prostate 2005;63:341-346.

10. Magi-Galluzzi C, Luo J, Isaacs WB, Hicks JL, de Marzo AM, Epstein JI. Alpha-methylacyl-CoA racemase: a variable sensitive immunohistochemical marker for the diagnosis of small prostate cancer foci on needle biopsy. Am $\mathrm{J}$ Surg Pathol 2003; 27:1128-1133.

11. Farinola MA, Epstein JI. Utility of immunohistochemistry for alpha-methylacyl-CoA racemase in distinguishing atrophic prostate cancer from benign atrophy. Hum Pathol 2004; 35:1272-1278.

12. Montironi R, Mazzucchelli R, Stramazzotti D, Scarpelli M, López-Beltrán A, Bostwick D. Basal cell hyperplasia and basal cell carcinoma of the prostate: a comprehensive review and discussion of a case with c-erbB-2 expression. J Clin Pathol 2005;58:290-296.

13. Rehman I, Cross SS, Azzouzi AR, Catto JW, Deloulme JC, Larre S, et al. S100A6 (Calcyclin) is a prostate basal cell marker absent in prostate cancer and its precursors. Br J Cancer 2004;91:739-744.

14. Xi Z, Klokk TI, Korkmaz K, Kurys P, Elbi C, Risberg B, et al. Kallikrein 4 is a predominantly nuclear protein and is overexpressed in prostate cancer. Cancer Res 2004; 64:2365-2370.

15. Xu J, Stolk JA, Zhang X, et al. Identification of differentially expressed genes in human prostate carcinoma using substraction and microarray. Am J Surg Pathol 2001;25:1397-1404.

16. Hsu SM, Raine L, Fanger H. The use of antiavidin antibody and avidin-biotin-peroxidase complex in immunoperoxidase techniques. Am J Clin Pathol 1981;75:876-882.

17. Kyprianou N, Tu H, Jacobs SC. Apoptotic versus proliferative activities in human benign prostatic hyperplasia. Hum Pathol 1996;27:668-675. 
18. Haussler O, Epstein JI, Amin MB, Heitz PU, Hailemariam S. Cell proliferation, apoptosis, oncogene, and tumor suppressor gene status in adenosis with comparison to benign prostatic hyperplasia, prostatic intraepithelial neoplasia and cancer. Hum Pathol 1999;30:1077-1086.

19. Johnson MI, Robinson MC, Marsh C, Robson CN, Neal DE, Hamdy FC. Expression of Bcl-2, Bax, and p53 in highgrade prostatic intraepithelial neoplasia and localized prostate cancer: relationship with apoptosis and proliferation. Prostate 1998;37:223-229.

20. Baltaci S, Orhan D, Ozer G, Tolunay O, Gogous O. Bcl-2 proto-oncogene expression in low- and high-grade prostatic intraepithelial neoplasia. BJU Int 2000;85:155-159.

21. Wang W, Bergh A, Damber JE. Chronic inflammation in benign prostate hyperplasia is associated with focal upregulation of cyclooxygenase-2, Bcl-2, and cell proliferation in the glandular epithelium. Prostate 2004;61:60-72.

22. Gaudin PB, Epstein JI. Adenosis of the prostate. Histologic features in needle biopsy specimens. Am J Surg Pathol 1995; 19:737-747.

23. Bostwick DG, Brawer MK. Prostatic intra-epithelial neoplasia and early invasion in prostate cancer. Cancer 1987; 59:788-794.
24. Zhou M, Jiang Z, Epstein JI. Expression and diagnostic utility of alpha-methylacyl-CoA racemase (P504S) in foamy gland and pseudohyperplastic prostate cancer. Am J Surg Pathol 2003;27:772-778.

25. Magi-Galluzzi G, Luo J, Isaacs WB, Hicks JL, de Marzo AM, Epstein JI. Alpha-methylacyl-CoA racemase: a variably sensitive immunohistochemical marker for the diagnosis of small prostate cancer foci on needle biopsy. Am J Surg Pathol 2003;27:1128-1133.

\author{
Dr. D. Ramos Soler \\ Departamento de PatologíA \\ Facultad de Medicina \\ Universitat de Valencia. Estudi General. \\ Avda. Blasco Ibáñez, 17 \\ 46010 - Valencia \\ E-mail: david.ramos@uv.es
}

(Trabajo recibido el 31 de agosto 2005) 